\title{
Optimal Recovery of Holomorphic Functions from Inaccurate Information about Radial Integration
}

\author{
Arthur DeGraw \\ Department of Mathematics and Statistics, State University of New York at Albany, Albany, USA \\ Email: adegraw@albany.edu
}

Received April 30, 2012; revised August 20, 2012; accepted September 2, 2012

\begin{abstract}
This paper addresses the optimal recovery of functions from Hilbert spaces of functions on the unit disc. The estimation, or recovery, is performed from inaccurate information given by integration along radial paths. For a holomorphic function expressed as a series, three distinct situations are considered: where the information error in $L_{2}$ norm is bound by $\delta>0$ or for a finite number of terms the error in $l_{2}^{N}$ norm is bound by $\delta>0$ or lastly the error in the $j^{\text {th }}$ coefficient is bound by $\delta_{j}>0$. The results are applied to the Hardy-Sobolev and Bergman-Sobolev spaces.
\end{abstract}

Keywords: Approximation; Optimal Recovery; Holomorphic

\section{Introduction}

Let $W$ be a subset of a linear space $X$, let $Z$ be a normed linear space, and $T$ the linear operator $T: X \rightarrow Z$ that we are trying to recover on $W \subset X$ from given information. This information is provided by a linear operator $I: X \rightarrow Y$ where $Y$ is a normed linear space. For any $x \in X$ we know some $\tilde{y} \in Y$ that is near $I x$. That is, we know $\tilde{y} \in Y$ such that

$$
\|I x-\tilde{y}\|_{Y} \leq \delta
$$

for some $\delta>0$. The value $\tilde{y}$ is our inaccurate information. Now we try to approximate the value of $T x$ from $\tilde{y}$ using an algorithm or method, $m$. Define a method to be any mapping $m: Y \rightarrow Z$, and regard $m(\tilde{y})$ as the approximation to $T x$ from the information $\tilde{y} \in Y$. Our goal is to minimize the difference of $T x$ and $m(\tilde{y})$ in $Z$, i.e. minimize $\|T x-m(\tilde{y})\|_{Z}$. However, the size of $\|T x-m(\tilde{y})\|_{Z}$. varies since $\tilde{y}$ can be chosen to be any $\tilde{y} \in Y$ satisfying (1). Furthermore $I x$ varies depending on the $x \in W$ chosen. So the error of any single method is defined as the worst case error

$$
e(T, W, I, \delta, m)=\sup _{\substack{x \in W_{0}, y \in Y \\ \| I x-y}}\|T x-m(\tilde{y})\|_{Z} .
$$

Now the optimal error is that of the method with the smallest error. Thus the error of optimal recovery is defined as

$$
\begin{aligned}
E(T, W, Y) & =\inf _{m: Y \rightarrow Z \rightarrow} \sup _{\substack{x \in W, \tilde{y} \in Y \\
\| x-\bar{y} \mid \mathbb{Y} \leq \delta}}\|T x-m(\tilde{y})\|_{Z} \\
& =\inf _{m: Y \rightarrow Z} e(T, W, Y, m) .
\end{aligned}
$$

For the problems addressed in this paper, let $Y_{1}, \cdots, Y_{n}$ be linear spaces with semi-inner norms $\|\cdot\|_{Y_{k}}$ and $I_{k}: X \rightarrow Y_{k}$ linear operators, $I=\left(I_{1}, \cdots, I_{n}\right)$. We want to recover $T X$ for

$$
x \in W=W_{k}=\left\{x \in X:\left\|I_{j} x\right\|_{Y_{j}} \leq \delta_{j}, 1 \leq j \leq k, 0 \leq k \leq n\right\}
$$

(where if $k=0$ we let $W_{0}=X$ ), if we know the values $\tilde{y}_{j}$ satisfying $\left\|I_{j} X-\tilde{y}_{j}\right\|_{Y_{j}} \leq \delta_{j}$ for $j=k+1, \cdots, n$.

Define the extremal problem

$$
\|T x\|_{Z}^{2} \rightarrow \max , \quad\left\|I_{j} x\right\|_{Y_{j}}^{2} \leq \delta_{i}^{2}, j=1, \cdots, n, \quad x \in X .
$$

This problem is dual to (2).

\section{Construction of Optimal Method and Error}

The following results of G. G. Magaril-Il'yaev and K. Yu. Osipenko [1] are applied to several problems of optimal recovery.

Theorem 1: Assume that there exist $\hat{\lambda}_{j} \geq 0$, $j=1, \cdots, n$ such that the solution of the extremal problem

$$
\|T x\|_{Z}^{2} \rightarrow \max , \quad \sum_{j=1}^{n} \hat{\lambda}_{j}\left\|I_{j} x\right\|_{Y_{j}}^{2} \leq \sum_{j=1}^{n} \hat{\lambda}_{j} \delta_{j}^{2}, \quad x \in X
$$


is the same as in (3). Assume also that for each $y=\left(y_{1}, \cdots, y_{n}\right) \in Y_{1} \times \cdots \times Y_{n}$ there exists $x_{y}=x\left(y_{1}, \cdots, y_{n}\right)$ which is a solution to

$$
\sum_{j=1}^{n} \hat{\lambda}_{j}\left\|I_{j} x-y_{j}\right\|_{Y_{j}}^{2} \rightarrow \min \quad x \in X
$$

Then for all $k, 0 \leq k<n$,

$$
E\left(T, W_{k}, I, \delta\right)=\sup _{\substack{x \in X \\\left\|I_{j} x\right\|_{Y_{j}} \leq \delta_{j}, j=1, \cdots, n}}\|T x\|_{Z}
$$

and the method

$$
\hat{m}\left(y_{k+1}, \cdots, y_{n}\right)=\operatorname{Tx}\left(0, \cdots, 0, y_{k+1}, \cdots, y_{n}\right)
$$

is optimal.

Theorem 1 gives a constructive approach to finding an optimal method $\hat{m}$ from the information. It follows from results obtained in [1-7] (see also [8] where this theorem was proven for one particular case.)

In order to apply Theorem 1 the values of extremal problems (4) and the dual problem (3) must agree. The following result, also due to G. G. Magaril-Il'yaev and K. $\mathrm{Yu}$ Osipenko [1], provides conditions under which the solution of problems (3) and (4) will agree.

Typically, when one encounters extremal problems, one approach is to construct the Lagrange function $\mathcal{L}$. For an extremal problem of the form of (4), the corresponding Lagrange function is

$$
\mathcal{L}\left(x, \lambda_{1}, \cdots, \lambda_{n}\right)=-\|T x\|_{Z}^{2}+\sum_{j=1}^{n} \lambda_{j}\left\|I_{j} x\right\|_{Y_{j}}^{2} .
$$

Furthermore, $\hat{x} \in X$ is called an extremal element if $\left\|I_{j} \hat{x}\right\|_{Y_{j}}^{2} \leq \delta_{j}^{2}$ for $j=1, \cdots, n$ and thus admissible in (4) and

$$
\|T \hat{x}\|_{Z}^{2}=\sup _{\substack{x \in X \\\left\|I_{j} x\right\|_{Y_{j}}^{2} \leq \delta_{j}^{2}, j=1, \cdots, n .}}\|T x\|_{Z}^{2} .
$$

Theorem 2: Let $\hat{\lambda}_{j} \geq 0$ and $\hat{x} \in X \quad$ be such that $\left\|I_{j} \hat{x}\right\|_{Y_{j}}^{2} \leq \delta_{j}^{2}$ for $1 \leq j \leq n$ and

1) $\min _{x \in X} \mathcal{L}\left(x, \hat{\lambda}_{1}, \cdots, \hat{\lambda}_{n}\right)=\mathcal{L}\left(\hat{x}, \hat{\lambda}_{1}, \cdots, \hat{\lambda}_{n}\right)$

2) $\sum_{j=1}^{n} \hat{\lambda}_{j}\left(\left\|I_{j} \hat{x}\right\|_{Y_{j}}^{2}-\delta_{j}^{2}\right)=0$.

Then $\hat{x}$ is an extremal element and

$$
\sup _{\substack{x \in X \\\left\|I_{j} x\right\|_{Y_{j}}^{2} \leq \delta_{j}^{2}, j=1, \cdots, n}}\|T x\|_{Z}^{2}=\sup _{\substack{x \in X \\ \sum_{j=1}^{n} \hat{\lambda}_{j}\left\|_{j}\right\|_{j} \|_{Y_{j}}^{2} \leq \sum_{j=1}^{n} \hat{\lambda}_{j} \delta_{j}^{2}}}\|T x\|_{Z}^{2}=\sum_{j=1}^{n} \hat{\lambda}_{j} \delta_{j}^{2} .
$$

If we wish to combine Theorems 1 and 2 to determine an optimal error and method then we must show the posed problem is able to satisfy equating extremal pro- blems (3) and (4). Through Theorem 2 we have such a means available.

\section{Main Results}

Consider the class of functions defined on the unit disc $\mathbb{D}=\{z:|z| \leq 1\}$ given by

$$
X=X_{\gamma}=\left\{f(z)=\sum_{j=0}^{\infty} a_{j} z^{j}: \sum_{j=0}^{\infty} \gamma_{j}\left|a_{j}\right|^{2}<\infty\right\}
$$

for $a_{j} \in \mathbb{C}, \gamma_{j} \geq 0$ satisfying

$$
\liminf _{j \rightarrow \infty} \gamma_{j}^{1 / j} \geq 1
$$

and

$$
\lim _{j \rightarrow \infty}\left(\gamma_{j}(j+1)\right)^{-1}=0 .
$$

Therefore, any $f \in X$ is holomorphic in the unit disc by (6). We define the semi-norm in $X$ as

$$
\|f\|_{X}=\left(\sum_{j=0}^{\infty} \gamma_{j}\left|a_{j}\right|^{2}\right)^{1 / 2}
$$

and

$$
W=\left\{f \in X:\|f\|_{X} \leq 1\right\} .
$$

Let $K: X \rightarrow L_{2}(\mathbb{T}), \mathbb{T}=[-\pi, \pi]$, be a linear operator given by

$$
K f(\phi)=\int_{0}^{1} f\left(r \mathrm{e}^{\mathrm{i} \phi}\right) \mathrm{d} r .
$$

That is, $K f$ is the radial integral of $f$. To see that $K f \in L_{2}(\mathbb{T})$, by (7) we have for all but finitely many $j$, $\gamma_{j} \geq \frac{c}{(j+1)^{2}}$ for some $c>0$. Thus if $\|f\|_{X}<\infty$ then $\|K f\|_{L_{2}(\mathbb{T})}<\infty$.

We assume to know $K f(\phi)$ given with a level of accuracy. That is, for a given $\delta>0$, we know a $\tilde{y} \in L_{2}(\mathbb{T})$ such that

$$
\|K f-\tilde{y}\|_{L_{2}(\mathbb{T})} \leq \delta .
$$

The problem of optimal recovery is to find an optimal recovery method of the function $f$ in the class $W$ from the information $\tilde{y}$ satisfying (9). The error of a given method is measured in the $L_{2}(\mathbb{D})$ norm defined by

$$
\|f\|_{L_{2}(\mathbb{D})}=\left(\frac{1}{2 \pi} \int_{0}^{1} \int_{-\pi}^{\pi}\left|f\left(r \mathrm{e}^{\mathrm{i} \phi}\right)\right|^{2} r \mathrm{~d} \phi \mathrm{d} r\right)^{1 / 2} .
$$

Any method $m: L_{2}(\mathbb{T}) \rightarrow L_{2}(\mathbb{D})$ is admitted as a recovery method. Let $\left\{\alpha_{j}\right\}_{j \in \mathbb{N}},\left\{\beta_{j}\right\}_{j \in \mathbb{N}}$ be sequences of non-negative real numbers such that 


$$
\lim _{j \rightarrow \infty} \beta_{j}=\infty, \quad \lim _{j \rightarrow \infty} \frac{\beta_{j}}{\alpha_{j}}=0 .
$$

Define $C=\operatorname{co}\left\{(0,0) \cup\left\{\left(\alpha_{j}, \beta_{j}\right)\right\}_{j \in \square}\right\}$ to be the convex hull. Define $\theta(x)$ for $x \in[0, \infty)$ by

$$
\theta(x)=\max \{y:(x, y) \in C\} \text {. }
$$

Lemma 1: The piecewise linear function $\theta$ with points of break $\left(x_{s}, y_{s}\right) \quad s=0,1, \cdots$, with $x_{0}<x_{1}<\cdots$, for $x \in\left[x_{s}, x_{s+1}\right]$ given by $\theta(x)=\lambda_{1} x+\lambda_{2}$ is such that $\lambda_{1}, \lambda_{2} \geq 0$.

Proof. Assume that $\lambda_{1}=\frac{y_{s+1}-y_{s}}{x_{s+1}-x_{s}}<0$. It means that $y_{s+1}<y_{s}$. Since $\beta_{i} \rightarrow \infty$ and $\alpha_{i} \rightarrow \infty$ as $j \rightarrow \infty$ there is a $k \in \mathbb{N}$ such that $\alpha_{k}>x_{s}$ and $\beta_{k}>y_{s}$. Then the interval between $\left(x_{s}, y_{s}\right)$ and $\left(\alpha_{k}, \beta_{k}\right)$ belongs to $C$. Consequently, $\theta\left(x_{s+1}\right)>y_{s+1}$ and $\left(x_{s+1}, y_{s+1}\right)$ is not a point of break of $\theta$.

Assume that $\lambda_{2}<0$. Since $(0,0) \in C$ the interval between $(0,0)$ and $\left(x_{s+1}, y_{s+1}\right)$ belongs to $C$. Geometrically, the line $(0,0)$ to $\left(x_{s+1}, y_{s+1}\right)$ will lie above the line $\theta(x)=\lambda_{1} x+\lambda_{2}$. It means that $\theta\left(x_{s}\right)>y_{s}$ contradicting that $\left(x_{s}, y_{s}\right)$ is a point of break of $\theta$.

Note that as $\lim _{j \rightarrow \infty} \frac{\beta_{j}}{\alpha_{j}}=0$ then for any fixed $\left(\alpha_{b}, \beta_{b}\right)$ the slopes between points $\left(\alpha_{b}, \beta_{b}\right)$ and $\left(\alpha_{j}, \beta_{j}\right)$ also tends to 0 as

$$
\lim _{j \rightarrow \infty} \frac{\beta_{j}-\beta_{b}}{\alpha_{j}-\alpha_{b}}=\lim _{j \rightarrow \infty} \frac{\frac{\beta_{j}}{\alpha_{j}}-\frac{\beta_{b}}{\alpha_{j}}}{\frac{\alpha_{j}}{\alpha_{j}}-\frac{\alpha_{b}}{\alpha_{j}}}=0 .
$$

\subsection{Inaccuracy in $L_{2}(\mathbb{T})$ Norm}

Consider the points in $\mathbb{R}^{2}$ given by $\left\{\left(\gamma_{j}(j+1)^{2}, j+1\right)\right\}_{j \in \mathbb{N}}$ and define the convex hull of the origin and this collection of points as $M$ :

$$
M=c o\left\{(0,0) \cup\left\{\left(\gamma_{j}(j+1)^{2}, j+1\right)\right\}_{j \in \square}\right\} .
$$

Let

$$
\theta(x)=\max \{y:(x, y) \in M\},
$$

thus $\theta$ is a piecewise linear function. Let $\left(x_{s}, y_{s}\right)$, $s=0,1, \cdots$ be the points of break of $\theta$ with $0=x_{0}<x_{1}<\cdots$. By (7) the assumption for Lemma 1 is satisfied by $\alpha_{j}=\gamma_{j}(j+1)^{2}$ and $\beta_{j}=j+1$.

Theorem 3: Suppose that $x_{s}<\delta^{-2} \leq x_{s+1}$ with $y_{s}>0$. Let

$$
\hat{\lambda}_{1}=\frac{y_{s+1}-y_{s}}{x_{s+1}-x_{s}}, \quad \hat{\lambda}_{2}=\frac{y_{s} x_{s+1}-y_{s+1} x_{s}}{x_{s+1}-x_{s}} .
$$

Then the error of optimal recovery is

$$
E(W, K, \delta)=\sqrt{\hat{\lambda}_{1}+\hat{\lambda}_{2} \delta^{2}}
$$

and

$$
\hat{m}(y)=\sum_{j=0}^{\infty}\left(1+\hat{\lambda}_{1} \hat{\lambda}_{2}^{-1} \gamma_{j}(j+1)^{2}\right)^{-1}(j+1) \tilde{y}_{j} z^{j}
$$

is an optimal method of recovery. If $y_{s}=0$ then $E(W, K, \delta)=\sqrt{\frac{y_{1}}{x_{1}}}$ and $\hat{m}(\tilde{y})=0$ is an optimal method.

Proof. Consider the dual extremal problem

$$
\|f\|_{L_{2}(\mathbb{D})}^{2} \rightarrow \max , \quad\|f\|_{X}^{2} \leq 1, \quad\|K f\|_{L_{2}(\mathbb{T})}^{2} \leq \delta^{2}
$$

which can be written as

$$
\begin{aligned}
& \sum_{j=0}^{\infty} \frac{1}{j+1}\left|a_{j}\right|^{2} \rightarrow \max \\
& \sum_{j=0}^{\infty} \gamma_{j}\left|a_{j}\right|^{2} \leq 1, \quad \sum_{j=0}^{\infty} \frac{1}{(j+1)^{2}}\left|a_{j}\right|^{2} \leq \delta^{2}
\end{aligned}
$$

where $f(z)=\sum_{j=0}^{\infty} a_{j} z^{j}$. Define the corresponding Lagrange function as

$$
\mathcal{L}\left(f, \lambda_{1}, \lambda_{2}\right)=\sum_{j=0}^{\infty} \frac{\left|a_{j}\right|^{2}}{(j+1)^{2}}\left(\lambda_{1} \gamma_{j}(j+1)^{2}+\lambda_{2}-(j+1)\right) .
$$

Let the line segment between successive points $\left(x_{s}, y_{s}\right)$ and $\left(x_{s+1}, y_{s+1}\right)$ be given by $\theta_{s}(x)=\hat{\lambda}_{1} x+\hat{\lambda}_{2}$. That is $\theta_{s}=\left.\theta\right|_{\left[x_{s}, x_{s+1}\right]}$. Thus $\hat{\lambda}_{1}, \hat{\lambda}_{2}$ are given by (12). Take any $\left(\gamma_{j}(j+1)^{2}, j+1\right)$, then by definition of the function $\theta$ we have

$$
j+1 \leq \theta\left(\gamma_{j}(j+1)^{2}\right) \leq \theta_{s}\left(\gamma_{j}(j+1)^{2}\right) .
$$

Thus for all $j=0,1, \cdots$

$$
j+1 \leq \hat{\lambda}_{1} \gamma_{j}(j+1)^{2}+\hat{\lambda}_{2}
$$

and hence $\mathcal{L}\left(f, \hat{\lambda}_{1}, \hat{\lambda}_{2}\right) \geq 0$ for any $f \in X$.

We proceed to the construction of a function $\hat{f}$ admissable in (15) that also satisfies $\hat{\lambda}_{1}\left(\|\hat{f}\|_{X}^{2}-1\right)+\hat{\lambda}_{2}\left(\|K \hat{f}\|_{L_{2}(\mathbb{T})}^{2}-\delta^{2}\right)=0$. Assume $y_{s}>0$. As $y_{s}=0$ if and only if $s=0$ and $y_{0}=0$ then $y_{s}>0$ if and only if $s>0$ or $y_{0}>0$. Let $k, k^{\prime} \in \mathbb{N}$ 
be the indices that satisfy

$$
\left(x_{s}, y_{s}\right)=\left(\gamma_{k}(k+1)^{2}, k+1\right)
$$

and

$$
\left(x_{s+1}, y_{s+1}\right)=\left(\gamma_{k^{\prime}}\left(k^{\prime}+1\right)^{2}, k^{\prime}+1\right) .
$$

We let $a_{j}=0$ for $j \neq k, k^{\prime}$, and choose $a_{k}, a_{k^{\prime}}$ so that they satisfy the conditions

$$
\begin{aligned}
& \gamma_{k}\left|a_{k}\right|^{2}+\gamma_{k^{\prime}}\left|a_{k^{\prime}}\right|^{2}=1 \\
& \frac{1}{(k+1)^{2}}\left|a_{k}\right|^{2}+\frac{1}{\left(k^{\prime}+1\right)^{2}}\left|a_{k^{\prime}}\right|^{2}=\delta^{2} .
\end{aligned}
$$

From these conditions let

$$
\begin{aligned}
a_{k} & =(k+1)\left(\frac{\delta^{2} \gamma_{k^{\prime}}\left(k^{\prime}+1\right)-1}{\gamma_{k^{\prime}}\left(k^{\prime}+1\right)^{2}-\gamma_{k}(k+1)^{2}}\right)^{1 / 2} \\
& =y_{s}\left(\frac{\delta^{2} x_{s+1}-1}{x_{s+1}-x_{s}}\right)^{1 / 2} \\
a_{k^{\prime}} & =\left(k^{\prime}+1\right)\left(\frac{1-\delta^{2} \gamma_{k}(k+1)^{2}}{\gamma_{k^{\prime}}\left(k^{\prime}+1\right)^{2}-\gamma_{k}(k+1)^{2}}\right)^{1 / 2} \\
& =y_{s+1}\left(\frac{1-\delta^{2} x_{s}}{x_{s+1}-x_{s}}\right)^{1 / 2}
\end{aligned}
$$

and

$$
\hat{f}(z)=a_{k} z^{k}+a_{k^{\prime}} z^{k^{\prime}}
$$

Now if $x_{s}<\delta^{-2} \leq x_{s+1}$ with $s>0$ or $s=0$ and $y_{0}>0$ the function $\hat{f}$ is admissible in (15) and $\mathcal{L}\left(\hat{f}, \hat{\lambda}_{1}, \hat{\lambda}_{2}\right)=0$, that is $\hat{f}$ minimizes $\mathcal{L}\left(f, \hat{\lambda}_{1}, \hat{\lambda}_{2}\right)$ and condition 1) of Theorem 2 is satisfied. Furthermore, by construction, $\hat{f}$ satisfies condition 2) of Theorem 2 .

If $y_{s}=0$, that is $s=0$ and $y_{0}=0$, define $a_{k^{\prime}}$ as in (17). Then as $x_{0}=0$

$$
a_{k^{\prime}}=y_{1}\left(\frac{1-\delta^{2} x_{0}}{x_{1}-x_{0}}\right)^{1 / 2}=y_{1} x_{1}^{-1 / 2}=\gamma_{k^{\prime}}^{-1 / 2} .
$$

So let $\hat{f}(z)=a_{k^{\prime}} z^{k^{\prime}}$ and we have

$$
\|\hat{f}\|_{X}^{2}=1, \quad\|K \hat{f}\|_{L_{2}(\mathbb{T})}^{2}=\frac{1}{\gamma_{k^{\prime}}\left(k^{\prime}+1\right)^{2}}=\frac{1}{x_{1}} \leq \delta^{2} .
$$

Thus the function $\hat{f}$ is admissable in (15) and satisfies 1) and 2) of Theorem 2. It should be noted that in this case $\hat{\lambda}_{1}, \hat{\lambda}_{2}$ are simply $\hat{\lambda}_{1}=y_{1} / x_{1}$ and $\hat{\lambda}_{2}=0$.

Now we proceed to the extremal problem

$$
\begin{aligned}
& \hat{\lambda}_{2}\|K f-\tilde{y}\|_{L_{2}(\mathbb{T})}^{2}+\hat{\lambda}_{1}\|f\|_{X}^{2} \rightarrow \min , \\
& f \in W, \tilde{y} \in L_{2}(\mathbb{T}) .
\end{aligned}
$$

This problem may be rewritten as

$$
\hat{\lambda}_{2} \sum_{-\infty}^{-1}\left|\tilde{y}_{j}\right|^{2}+\hat{\lambda}_{2} \sum_{j=0}^{\infty}\left|\frac{1}{j+1} a_{j}-\tilde{y}_{j}\right|^{2}+\hat{\lambda}_{1} \sum_{j=0}^{\infty} \gamma_{j}\left|a_{j}\right|^{2} \rightarrow \min
$$

which has solution

$$
a_{j}=\left\{\begin{array}{cc}
\frac{j+1}{1+\hat{\lambda}_{1} \hat{\lambda}_{2}^{-1} \gamma_{j}(j+1)^{2}} \tilde{y}_{j}: j \geq 0, \hat{\lambda}_{2} \neq 0 \\
0 & : \text { otherwise }
\end{array}\right.
$$

So for $x_{s}<\delta^{-2} \leq x_{s+1}, \quad y_{s}>0$ by Theorems 1 and 2, (14) is an optimal method and the error of optimal recovery is given by (13). If $y_{s}=0$ then

$E(W, K, \delta)=\sqrt{\frac{y_{1}}{x_{1}}}$ and $\hat{m}(\tilde{y})=0$ is an optimal method.

It should be noted that for fixed $\hat{\lambda}_{1}, \hat{\lambda}_{2} \neq 0$, that is for a fixed $\delta>0$, the terms

$$
\varepsilon_{j}=\left(1+\hat{\lambda}_{1} \hat{\lambda}_{2}^{-1} \gamma_{j}(j+1)^{2}\right)^{-1}(j+1)
$$

will have the property, $0<\varepsilon_{j}<1$ and $\lim _{j \rightarrow \infty} \varepsilon_{j}=0$ as $\lim _{j \rightarrow \infty}\left(\gamma_{j}(j+1)\right)^{-1}=0$. So $\hat{m}$ smooths approximate values of the coefficients of $\tilde{y}$ by the filter $\varepsilon_{j}$.

\subsection{Inaccuracy in $I_{2}^{N}$ Norm}

Our next problem of optimal recovery remains to recover $f \in X=X_{\gamma}$ from inaccurate information pertaining to the radial integral of $f$. However, the inaccurate information we are given are the values

$\tilde{y}_{0}, \tilde{y}_{1}, \cdots, \tilde{y}_{N-1} \in \square$ such that

$$
\sum_{j=0}^{N-1}\left|K_{j} f-\tilde{y}_{j}\right|^{2} \leq \delta^{2}
$$

where $K_{j} f$ is the $j^{\text {th }}$ coefficient of the radial integral Kf ,

$$
K_{j} f=\left\langle K f(\phi), \mathrm{e}^{-\mathrm{ij} \phi}\right\rangle_{L_{2}(\mathbb{T})} .
$$

Denote

$$
K^{N}=\left(K_{0}, K_{1}, \cdots, K_{N-1}\right) .
$$

We again consider the space of functions $X=X_{\gamma}$ given by (5) and $M$ and $\theta$ defined by (10) and (11) respectively but now add the condition

$$
\gamma_{j}>0, \quad j \geq N
$$

The problem of optimal recovery on the class $W$ 
given by (8) is to determine the optimal error

$$
\begin{aligned}
& E\left(W, K^{N}, \delta\right)= \\
& \inf _{m: \square^{N} \rightarrow L_{2}(\mathbb{D})} \sup _{f \in W,} \sum_{\substack{\left.N-1 \\
\sum_{j=0} \mid \tilde{y}_{0}, \tilde{y}_{1}, \cdots, \tilde{y}_{N-1}\right)\left.\in \tilde{y}_{j}\right|^{2} \leq \delta^{2}}}\|f-m(\tilde{y})\|_{L_{2}(\mathbb{D})}
\end{aligned}
$$

and an optimal method $\hat{m}: \mathbb{C}^{N} \rightarrow L_{2}(\mathbb{D})$ obtaining this error.

Define $l_{0} \in \mathbb{N}$ as the largest index such that

$$
\left(\gamma_{l_{0}}\left(l_{0}+1\right)\right)^{-1}=\max _{l \geq N}\left\{\left(\gamma_{l}(l+1)\right)^{-1}\right\},
$$

which by (7) exists, and

$$
s_{0}=\min \left\{s \geq 0:\left(\gamma_{l_{0}}\left(l_{0}+1\right)\right)^{-1} \geq \frac{y_{s+1}-y_{s}}{x_{s+1}-x_{s}}\right\} .
$$

Theorem 4: Suppose $x_{s}<\delta^{-2} \leq x_{s+1}$ with $s<s_{0}$. If $y_{s}>0$ let $\hat{\lambda}_{1}=\frac{y_{s+1}-y_{s}}{x_{s+1}-x_{s}}$ and $\hat{\lambda}_{2}=\frac{y_{s} x_{s+1}-y_{s+1} x_{s}}{x_{s+1}-x_{s}}$.

Then the optimal error is

$$
E\left(W, K^{N}, \delta\right)=\sqrt{\hat{\lambda}_{1}+\hat{\lambda}_{2} \delta^{2}}
$$

and

$$
\hat{m}(\tilde{y})=\sum_{j=0}^{N-1}\left(1+\hat{\lambda}_{1} \hat{\lambda}_{2}^{-1} \gamma_{j}(j+1)^{2}\right)^{-1}(j+1) \tilde{y}_{j} z^{j}
$$

is an optimal method. If $y_{s}=0$ then

$E\left(W, K^{N}, \delta\right)=\sqrt{\frac{y_{1}}{x_{1}}}$ and $\hat{m}(\tilde{y})=0$ is an optimal method.

If $\delta^{-2}>x_{s_{0}}$ and $y_{s_{0}}>0$ then with

$\hat{\lambda}_{1}=\left(\gamma_{l_{0}}\left(l_{0}+1\right)\right)^{-1}$ and $\hat{\lambda}_{2}=y_{s_{0}}-x_{s_{0}} \hat{\lambda}_{1}$ the error of optimal recovery is (22) and (23) is an optimal method. For $y_{s_{0}}=0, E\left(W, K^{N}, \delta\right)=\sqrt{\frac{y_{1}}{x_{1}}}$ and $\hat{m}(\tilde{y})=0$ is an optimal method.

Proof. For the cases $x_{s}<\delta^{-2} \leq x_{s+1}$ with $s<s_{0}$ we simply apply the same structure of proof as in Theorem 3 . For the case $\delta^{-2}>x_{s_{0}}$ there remains some work.

Our construction will depend on whether or not $y_{s_{0}}=0$, that is whether or not $s_{0}=0$ with $\left(x_{s_{0}}, y_{s_{0}}\right)=(0,0)$.

First we notice $y_{s_{0}} \leq N$. Assume not. Then if $y_{s_{0}}>N>0$ we also know $x_{s_{0}}>0$ since for all $j \geq N$ we assumed $\gamma_{j}>0$. Since $x_{0}=0$ we know $s_{0}>0$. Then by definition of $s_{0}$ we know for $0 \leq s<s_{0}$,

$$
\frac{y_{s+1}-y_{s}}{x_{s+1}-x_{s}}>\left(\gamma_{l_{0}}\left(l_{0}+1\right)\right)^{-1}
$$

and substituting $s=s_{0}-1$ we have

$$
\frac{y_{s_{0}}}{x_{s_{0}}} \geq \frac{y_{s_{0}}-y_{s_{0}-1}}{x_{s_{0}}-x_{s_{0}-1}}>\left(\gamma_{l_{0}}\left(l_{0}+1\right)\right)^{-1}
$$

which contradicts the definition of $l_{0}$. Therefore $y_{s_{0}} \leq N$ and if $\left(x_{s_{0}}, y_{s_{0}}\right)=\left(\gamma_{c}(c+1)^{2}, c+1\right)$ then $c<N$.

In either case, $y_{s_{0}}=0$ or $y_{s_{0}}>0$, the dual problem is of the form

$$
\|f\|_{L_{2}(\mathbb{D})}^{2} \rightarrow \max
$$

$$
\|f\|_{X}^{2} \leq 1, \quad \sum_{j=0}^{N-1}\left|K_{j} f\right|^{2} \leq \delta^{2}, \quad f \in X .
$$

The corresponding Lagrange function is then

$$
\mathcal{L}\left(f, \lambda_{1}, \lambda_{2}\right)=\sum_{j=0}^{\infty} \frac{\left|a_{j}\right|^{2}}{(j+1)^{2}}\left(\lambda_{1} \gamma_{j}(j+1)^{2}+\chi_{j}^{N} \lambda_{2}-(j+1)\right)
$$

where $\chi_{j}^{N}$ is the characteristic function of $\{j \in \square: j<N\}$.

Case 1): $y_{s_{0}}>0$

If $y_{s_{0}}>0$ let $c$ correspond to the index satisfying

$$
\left(x_{s_{0}}, y_{s_{0}}\right)=\left(\gamma_{c}(c+1)^{2}, c+1\right) \text {. }
$$

To determine $\hat{\lambda}_{1}, \hat{\lambda}_{2}$ let $y=\hat{\lambda}_{1} x+\hat{\lambda}_{2}$ be the line through the point $\left(x_{s_{0}}, y_{s_{0}}\right)$ that is parallel to the line from the origin to $\left(\gamma_{l_{0}}\left(l_{0}+1\right)^{2}, l_{0}+1\right)$. That is, let

$$
\hat{\lambda}_{1}=\left(\gamma_{l_{0}}\left(l_{0}+1\right)\right)^{-1}, \hat{\lambda}_{2}=y_{s_{0}}-x_{s_{0}} \hat{\lambda}_{1}
$$

So for any point of break we have $y_{s} \leq \hat{\lambda}_{1} x_{s}+\hat{\lambda}_{2}$ and for any index $j \leq N-1$, we obtain

$$
j+1 \leq \theta\left(\gamma_{j}(j+1)^{2}\right) \leq \hat{\lambda}_{1} \gamma_{j}(j+1)^{2}+\hat{\lambda}_{2} .
$$

If $j \geq N$ then

$$
\begin{aligned}
\hat{\lambda}_{1} \gamma_{j}(j+1)-1 & =\left(\gamma_{l_{0}}\left(l_{0}+1\right)\right)^{-1} \gamma_{j}(j+1)-1 \\
& \geq\left(\gamma_{j}(j+1)\right)^{-1} \gamma_{j}(j+1)-1 \\
& =0 .
\end{aligned}
$$

Thus for the chosen $\hat{\lambda}_{1}$ and $\hat{\lambda}_{2}$ and any $f \in X$ we have $\mathcal{L}\left(f, \hat{\lambda}_{1}, \hat{\lambda}_{2}\right) \geq 0$.

To construct $\hat{f} \in X$ admissable in (24), let $\hat{a}_{j}=0$ for $j \neq c, l_{0}$ and define $\hat{a}_{c}, \hat{a}_{l_{0}}$ by the system

$$
\|\hat{f}\|_{X}^{2}=1, \quad \sum_{j=0}^{N-1}\left|K_{j} \hat{f}\right|^{2}=\delta^{2}
$$

and since $0 \leq c<N \leq l_{0}$ this becomes 


$$
\gamma_{c}\left|\hat{a}_{c}\right|^{2}+\gamma_{l_{0}}\left|\hat{a}_{l_{0}}\right|^{2}=1, \frac{\left|\hat{a}_{c}\right|^{2}}{(c+1)^{2}}=\delta^{2} .
$$

So let $\hat{a}_{c}=\delta(c+1)$ and $\hat{a}_{l_{0}}=\left(\frac{1-\delta^{2} \gamma_{c}(c+1)^{2}}{\gamma_{l_{0}}}\right)^{1 / 2}$. Then for $\delta^{-2} \geq \gamma_{c}(c+1)^{2}=x_{s_{0}}$ the function $\hat{f}(z)=\hat{a}_{c} z^{c}+\hat{a}_{l_{0}} z^{l_{0}} \quad$ is admissable in (24) with

$$
\mathcal{L}\left(\hat{f}, \hat{\lambda}_{1}, \hat{\lambda}_{2}\right)=0
$$

Therefore $\mathcal{L}\left(\hat{f}, \hat{\lambda}_{1}, \hat{\lambda}_{2}\right)=\min _{f \in X} \mathcal{L}\left(f, \hat{\lambda}_{1}, \hat{\lambda}_{2}\right)$ and by construction we have $\|\hat{f}\|_{X}^{2}=1$ and $\sum_{j=0}^{N-1}\left|K_{j} \hat{f}\right|^{2}=\delta^{2}$ so that

$$
\hat{\lambda}_{1}\left(\|\hat{f}\|_{X}^{2}-1\right)+\hat{\lambda}_{2}\left(\sum_{j=0}^{N-1}\left|K_{j} \hat{f}\right|^{2}-\delta^{2}\right)=0
$$

and conditions (a) and (b) of Theorem 2 are satisfied.

Case 2): $y_{s_{0}}=0$

If $y_{s_{0}}=0$ then $\left(x_{s_{0}}, y_{s_{0}}\right)=(0,0)$, and $s_{0}=0$, as this is the only point in the set

$$
\left\{(0,0) \cup\left\{\left(\gamma_{j}(j+1)^{2}, j+1\right)\right\}_{j \in \mathbb{N}}\right\} \text { with a } y \text {-coordinate }
$$

of 0 . Furthermore, as $(0,0)$ is a point of break of $\theta$ we know $\gamma_{j}>0$ for all $j \in \mathbb{N}$. Since $s_{0}=0$ then by the definition of $s_{0}$ we know $\frac{y_{1}}{x_{1}} \leq\left(\gamma_{l_{0}}\left(l_{0}+1\right)\right)^{-1}$. As

$$
\begin{aligned}
\frac{y_{1}}{x_{1}} & =\max _{j \geq 0}\left\{\left(\gamma_{j}(j+1)\right)^{-1}\right\} \\
& \geq \max _{j \geq N}\left\{\left(\gamma_{j}(j+1)\right)^{-1}\right\}=\left(\gamma_{l_{0}}\left(l_{0}+1\right)\right)^{-1}
\end{aligned}
$$

then we obtain equality, $\frac{y_{1}}{x_{1}}=\left(\gamma_{l_{0}}\left(l_{0}+1\right)\right)^{-1}$.

Define $\hat{\lambda}_{1}, \hat{\lambda}_{2}$ by (25) so $\hat{\lambda}_{1}=\frac{y_{1}}{x_{1}}$ and $\hat{\lambda}_{2}=0$. If we let $\hat{f} \in X$ be $\hat{f}(z)=\left(\gamma_{l_{0}}\right)^{-1 / 2} z^{l_{0}}$ then

$$
\begin{aligned}
& \hat{\lambda}_{1}\left(\|\hat{f}\|_{X}^{2}-1\right)+\hat{\lambda}_{2}\left(\sum_{j=0}^{N-1}\left|K_{j} \hat{f}\right|^{2}-\delta^{2}\right) \\
& =\hat{\lambda}_{1}(1-1)+0\left(0-\delta^{2}\right)=0 .
\end{aligned}
$$

In addition $\hat{f}$ is admissable in extremal problem (24) as $\|\hat{f}\|_{X}^{2} \leq 1$ and $\sum_{j=0}^{N-1}\left|K_{j} \hat{f}\right|^{2}=0 \leq \delta^{2}$.

To justify $\mathcal{L}\left(f, \hat{\lambda}_{1}, \hat{\lambda}_{2}\right) \geq 0$ simply note that as $\left(x_{1}, y_{1}\right)$ satisfies (26) and $\gamma_{j}>0$ for all $j \geq 0$ then $\frac{y_{1}}{x_{1}} \gamma_{j}(j+1)-1 \geq 0$. So we have

$\mathcal{L}\left(f, \hat{\lambda}_{1}, \hat{\lambda}_{2}\right)=\sum_{j=0}^{\infty} \frac{\left|a_{j}\right|^{2}}{j+1}\left(\frac{y_{1}}{x_{1}} \gamma_{j}(j+1)-1\right) \geq 0$. Since $\mathcal{L}\left(f, \hat{\lambda}_{1}, \hat{\lambda}_{2}\right)=0$ then $\hat{f}$ minimizes $\mathcal{L}$.

For both cases, we now consider extremal problem

$$
\hat{\lambda}_{1}\|f\|_{X}^{2}+\hat{\lambda}_{2} \sum_{j=0}^{N-1}\left|K_{j} f-\tilde{y}_{j}\right|^{2} \rightarrow \min , \quad f \in X .
$$

This problem can be written as

$$
\hat{\lambda}_{1} \sum_{j=0}^{\infty} \gamma_{j}\left|a_{j}\right|^{2}+\hat{\lambda}_{2} \sum_{j=0}^{N-1}\left|\frac{a_{j}}{j+1}-\tilde{y}_{j}\right|^{2} \rightarrow \min ,
$$

which will have solution

$$
a_{j}=\left\{\begin{array}{cc}
\frac{j+1}{1+\hat{\lambda}_{1} \hat{\lambda}_{2}^{-1} \gamma_{j}(j+1)^{2}} \tilde{y}_{j}: 0 \leq j \leq N-1, \hat{\lambda}_{2} \neq 0 \\
0 \quad: j \geq N, \text { or } \hat{\lambda}_{2}=0
\end{array}\right.
$$

So by Theorems 1 and 2 we have obtained the optimal error and an optimal method for all scenarios. In each case $i$ and ii, $\hat{\lambda}_{1}$ and $\hat{\lambda}_{2}$ are given by (25). In each case, the error of optimal recovery is

$E\left(W, K^{N}, \delta\right)=\sqrt{\hat{\lambda}_{1}+\hat{\lambda}_{2} \delta^{2}}$ which for case 2) simplifies to $E\left(W, K^{N}, \delta\right)=\sqrt{\frac{y_{1}}{x_{1}}}$. Also for each case, a method of optimal recovery is given by $\hat{m}(\tilde{y})=\sum_{j=0}^{N-1} a_{j} z^{j}$ where in case 2) this simplifies to $\hat{m}(\tilde{y})=0$ since in case 2), $\hat{\lambda}_{2}=0$.

One may be able to reduce the amount information needed without affecting the error of optimal recovery. Therefore, by reducing the number of terms in the optimal method we reduce the compututaions needed. The following ideas are in [9]. We consider the subset $J_{s} \subseteq C, s<s_{0}$ as the set of all points whose slope to the origin is greater than the slope of $\theta(x)$ for $x \in\left[x_{s}, x_{s+1}\right]$, that is the slope of the line segment between points $\left(x_{s}, y_{s}\right)$ and $\left(x_{s+1}, y_{s+1}\right)$. Define the sets

$$
J_{s}=\left\{0 \leq j \leq N-1:\left(\gamma_{j}(j+1)\right)^{-1} \geq \frac{y_{s+1}-y_{s}}{x_{s+1}-x_{s}}\right\},
$$

for $s=0,1, \cdots, s_{0}$ where if $\gamma_{j}=0$ define $\left(\gamma_{j}(j+1)\right)^{-1}=\infty$. Now consider the same problem as stated in Theorem 4 using only information $K^{J_{s}} f$. For $y_{s}>0$, we have $\frac{y_{s}}{x_{s}}>\frac{y_{s+1}}{x_{s+1}} \geq \frac{y_{s+1}-y_{s}}{x_{s+1}-x_{s}}$ and so 
$\left(x_{s}, y_{s}\right),\left(x_{s+1}, y_{s+1}\right) \in\left\{\left(\gamma_{j}(j+1)^{2}, j+1\right)\right\}_{j \in J_{s}}$. In this situation, $0 \leq s<s_{0}$ with $y_{s}>0$, it was shown that the error of optimal recovery only involves the two points $\left(x_{s}, y_{s}\right),\left(x_{s+1}, y_{s+1}\right)$ then the reduction in information from $K^{N}$ to $K^{J_{s}}$ will not change the error. That is $E\left(W, K^{N}, \delta\right)=E\left(W, K^{J_{s}}, \delta\right)$ and if $\left|J_{s}\right|=\tilde{N}$, an optimal method is

$$
\hat{m}(y)=\sum_{k=0}^{\tilde{N}}\left(1+\hat{\lambda}_{1} \hat{\lambda}_{2} \gamma_{j_{k}}\left(j_{k}+1\right)^{2}\right)^{-1}\left(j_{k}+1\right) y_{j_{k}} z^{j_{k}}
$$

where $y=\left(y_{j_{0}}, \cdots, y_{j_{\tilde{N}}}\right)$.

\subsection{Varying Levels of Accuracy Termwise}

In Theorems 3 and 4 the inaccuracy of the information given is a total inaccuracy. That is, the inaccuracy $\delta^{2}$ is an upper bound on the sum total of the inaccuracies in each term, be it a finite or infinite sum. For Theorems 3 and 4 however, there is no way to tell how the inaccuracy is distributed. In particular, with regards to Theorem 4, the situations in which the given information $\tilde{y}=\left(\tilde{y}_{0}, \cdots, \tilde{y}_{N-1}\right)$ satisfies

$$
\left|K_{j} f-\tilde{y}_{j}\right| \leq \delta / \sqrt{N}, \quad j=0, \cdots, N-1
$$

or for some particular $m \in \mathbb{N}$ satisfying $0 \leq m \leq N-1$

$$
\left|K_{j} f-\tilde{y}_{j}\right| \leq\left\{\begin{array}{l}
\delta: j=m \\
0: j \neq m
\end{array}\right.
$$

are treated the same. For the next problem of optimal recovery we address this ambiguity. The problem of optimal recovery is to determine an optimal method and the optimal error of recovering $f \in X_{\gamma}$, from the information $\tilde{y}=\left(\tilde{y}_{0}, \cdots, \tilde{y}_{N-1}\right) \in \mathbb{C}^{N}$ satisfying

$$
\left|K_{j} f-\tilde{y}_{j}\right| \leq \delta_{j}
$$

for some prescribed $\delta_{j} \geq 0$ and $j=0, \cdots, N-1$.

To define $X_{\gamma}$ use conditions (6) and (20) as previously but impose an additional restriction. We add the condition

$$
\gamma_{j}(j+1) \leq \gamma_{j+1}(j+2), \quad j=0,1, \cdots .
$$

Define $\bar{\delta}=\left(\delta_{0}, \cdots, \delta_{N-1}\right)$ where $\delta_{j} \geq 0$ are the levels of accuracy. If $\gamma_{0} \delta_{0}^{2} \leq 1$ define

$$
p_{0}=\max \left\{p \geq 0: \sum_{j=0}^{p} \delta_{j}^{2} \gamma_{j}(j+1)^{2} \leq 1, \quad p \leq N-1\right\} \text {. }
$$

So $p_{0} \geq 0$ and furthermore $\gamma_{p_{0}+1}>0$. The case $\gamma_{0} \delta_{0}^{2}>1$ will be treated seperately.

Theorem 5: If $\gamma_{0} \delta_{0}^{2} \leq 1$ let

$$
\begin{gathered}
\hat{\lambda}=\frac{1}{\gamma_{p_{0}+1}\left(p_{0}+2\right)} \\
\hat{\lambda}_{j}=\left\{\begin{array}{cc}
j+1-\hat{\lambda} \gamma_{j}(j+1)^{2} & : j \leq p_{0} \\
0 & : p_{0}+1 \leq j \leq N-1
\end{array}\right.
\end{gathered}
$$

then the error of optimal recovery is given by

$$
E\left(W, K^{N}, \bar{\delta}\right)=\sqrt{\hat{\lambda}+\sum_{j=0}^{p_{0}} \delta_{j}^{2} \hat{\lambda}_{j}}
$$

and

$$
\hat{m}(\tilde{y})=\sum_{j=0}^{p_{0}} \hat{\lambda}_{j} \tilde{y}_{j} z^{j}
$$

is an optimal method.

If $\gamma_{0} \delta_{0}^{2}>1$ then $E\left(W, K^{N}, \bar{\delta}\right)=\gamma_{0}^{-1 / 2}$ and $\hat{m}(\tilde{y})=0$ is an optimal method.

Proof. The dual problem in this situation is

$$
\begin{gathered}
\|f\|_{L_{2}(\mathbb{D})}^{2} \rightarrow \max \\
\|f\|_{X}^{2} \leq 1, \quad \frac{\left|a_{j}\right|^{2}}{(j+1)^{2}} \leq \delta_{j}^{2}, \quad j=0,1, \cdots, N-1
\end{gathered}
$$

with the corresponding Lagrange function

$$
\begin{aligned}
\mathcal{L}(f, \bar{\lambda})= & -\sum_{j=0}^{\infty} \frac{\left|a_{j}\right|^{2}}{j+1}+\lambda \sum_{j=0}^{\infty} \gamma_{j}\left|a_{j}\right|^{2}+\sum_{j=0}^{N-1} \lambda_{j} \frac{\left|a_{j}\right|^{2}}{(j+1)^{2}} \\
= & \sum_{j=0}^{N-1} \frac{\left|a_{j}\right|^{2}}{(j+1)^{2}}\left(\lambda \gamma_{j}(j+1)^{2}+\lambda_{j}-(j+1)\right) \\
& +\sum_{j=N}^{\infty} \frac{\left|a_{j}\right|^{2}}{(j+1)^{2}}\left(\lambda \gamma_{j}(j+1)^{2}-(j+1)\right)
\end{aligned}
$$

The method of proof will be to first determine $\overline{\hat{\lambda}}=\left(\hat{\lambda}, \hat{\lambda}_{1}, \cdots, \hat{\lambda}_{N-1}\right)$ with $\hat{\lambda}, \hat{\lambda}_{j} \geq 0$ and $\hat{f} \in X$ admissable in (31) and satisfying 1) and 2) of Theorem 2.

If $\gamma_{0} \delta_{0}^{2} \leq 1$, define $\hat{\lambda}$ and $\hat{f}$ as follows:

$$
\begin{gathered}
\hat{\lambda}=\frac{1}{\gamma_{p_{0}+1}\left(p_{0}+2\right)} \\
\hat{\lambda}_{j}=\left\{\begin{array}{cc}
j+1-\hat{\lambda} \gamma_{j}(j+1)^{2}: & j \leq p_{0} \\
0 & : p_{0}+1 \leq j \leq N-1
\end{array}\right. \\
\hat{a}_{j}=\left\{\begin{array}{cc}
\delta_{j}(j+1) & : j \leq p_{0} \\
\left(\left(1-\sum_{j=0}^{p_{0}} \gamma_{j} \delta_{j}^{2}(j+1)^{2}\right) \frac{1}{\gamma_{p_{0}+1}}\right)^{1 / 2}: j=p_{0}+1 \\
0 & : j>p_{0} .
\end{array}\right.
\end{gathered}
$$


To verify $\hat{\lambda}_{j} \geq 0$ assume $j \leq p_{0}$ in which case $\gamma_{j}(j+1)^{2} \leq \gamma_{p_{0}+1}\left(p_{0}+2\right)(j+1)$ and hence

$$
\begin{aligned}
\hat{\lambda}_{j} & =j+1-\frac{1}{\gamma_{p_{0}+1}\left(p_{0}+2\right)} \gamma_{j}(j+1)^{2} \\
& \geq j+1-\frac{1}{\gamma_{p_{0}+1}\left(p_{0}+2\right)} \gamma_{p_{0}+1}\left(p_{0}+2\right)(j+1)=0 .
\end{aligned}
$$

To show for the chosen $\hat{\bar{\lambda}}$ and any $f \in X$, $\mathcal{L}(f, \hat{\bar{\lambda}}) \geq 0$, we consider the cases $j \leq p_{0}$ or $j>p_{0}$. For $j>p_{0}$ we know by assumption $\gamma_{p_{0}+1}\left(p_{0}+2\right) \leq \gamma_{j}(j+1)$ and hence

$$
\begin{aligned}
\hat{\lambda} \gamma_{j}(j+1)-1 & =\frac{1}{\gamma_{p_{0}+1}\left(p_{0}+2\right)} \gamma_{j}(j+1)-1 \\
& \geq 1-1=0
\end{aligned}
$$

For $j \leq p_{0}$

$$
\begin{aligned}
& \hat{\lambda} \gamma_{j}(j+1)^{2}+\hat{\lambda}_{j}-(j+1) \\
& =\hat{\lambda} \gamma_{j}(j+1)^{2}+\left(j+1-\hat{\lambda} \gamma_{j}(j+1)^{2}\right)-(j+1)=0 .
\end{aligned}
$$

Thus for any $f \in X, \mathcal{L}(f, \overline{\hat{\lambda}}) \geq 0$. For the constructed $\hat{f}$, it can be shown that $\mathcal{L}(f, \overline{\hat{\lambda}})=0$ as desired. and thus $\hat{f}$ minimizes the Lagrange function.

To show $\hat{f}$ is admissable in (31) we can clearly see that for $j \leq p_{0}, \frac{\left|\hat{a}_{j}\right|^{2}}{(j+1)^{2}} \leq \delta_{j}^{2}$. It remains to show $\frac{\left|\hat{a}_{p_{0}+1}\right|^{2}}{\left(p_{0}+2\right)^{2}} \leq \delta_{p_{0}+1}^{2}$ for $p_{o}<N-1$. Assume not, then

$$
\left(\left(1-\sum_{j=0}^{p_{0}} \delta_{j}^{2} \gamma_{j}(j+1)^{2}\right) \frac{1}{\gamma_{p_{0}+1}\left(p_{0}+2\right)^{2}}\right)>\delta_{p_{0}+1}^{2}
$$

which occurs if and only if

$$
1>\sum_{j=0}^{p_{0}+1} \delta_{j}^{2} \gamma_{j}(j+1)^{2}
$$

which contradicts the definition of $p_{0}$ unless $p_{0}=N-1$. If $p_{0}=N-1$ then $p_{0}+1=N$ and hence we no longer need the condition $\frac{\left|\hat{a}_{p_{0}+1}\right|^{2}}{\left(p_{0}+2\right)^{2}} \leq \delta_{p_{0}+1}^{2}$ in order for $\hat{f}$ to satisfy (31).

Furthermore

$$
\begin{aligned}
& \|\hat{f}\|_{X}^{2}=\left(\sum_{j=0}^{p_{0}} \gamma_{j}\left|\hat{a}_{j}\right|^{2}\right)+\gamma_{p_{0}+1}\left|\hat{a}_{p_{0}+1}\right|^{2} \\
& =\left(\sum_{j=0}^{p_{0}} \gamma_{j}\left|\hat{a}_{j}\right|^{2}\right)+\left(1-\left(\sum_{j=0}^{p_{0}} \gamma_{j}\left|\hat{a}_{j}\right|^{2}\right)\right)=1
\end{aligned}
$$

and so $\hat{f}$ is admissable in (31).

By the construction of $\hat{f}$ we also have the results $\|\hat{f}\|_{X}=1$ and $\frac{\left|\hat{a}_{j}\right|^{2}}{(j+1)^{2}}=\delta_{j}^{2}$ for $j \leq p_{0}$ while $\hat{\lambda}_{j}=0$ for $j>p_{0}$. Thus $\hat{f}$ satisfies 2) of Theorem 2 as

$$
\hat{\lambda}\left(\|\hat{f}\|_{X}^{2}-1\right)+\sum_{j=0}^{N-1} \hat{\lambda}_{j}\left(\frac{\left|\hat{a}_{j}\right|^{2}}{(j+1)^{2}}-\delta_{j}^{2}\right)=0 .
$$

We now proceed to the extremal problem

$$
\hat{\lambda}\|f\|_{X}^{2}+\sum_{j=0}^{p_{0}} \hat{\lambda}_{j}\left|K_{j} f-\tilde{y}_{j}\right|^{2} \rightarrow \min , \quad f \in X .
$$

Notice the upper bound on the sum is $p_{0} \leq N-1$ as $\hat{\lambda}_{j}=0$ for any $j>p_{0}$. This extremal problem will have solution

$$
a_{j}=\left\{\begin{array}{cc}
\frac{\hat{\lambda}_{j}(j+1)}{\hat{\lambda} \gamma_{j}(j+1)^{2}+\hat{\lambda}_{j}} \tilde{y}_{j}=\hat{\lambda}_{j} \tilde{y}_{j}: j \leq p_{0} . \\
0 & : j>p_{0}
\end{array} .\right.
$$

Therefore the error of optimal recovery is given by

$$
E\left(W, K^{N}, \bar{\delta}\right)=\sqrt{\hat{\lambda}+\sum_{j=0}^{p_{0}} \delta_{j}^{2} \hat{\lambda}_{j}}
$$

and

$$
\hat{m}(\tilde{y})=\sum_{j=0}^{p_{0}} \hat{\lambda}_{j} \tilde{y}_{j} z^{j}
$$

is an optimal method.

Now we proceed to the case $\gamma_{0} \delta_{0}^{2}>1$. Choose $\hat{\lambda}=\gamma_{0}^{-1}$ and $\hat{\lambda}_{j}=0$ for $j=0,1, \cdots, N-1$. Then as $\gamma_{0} \leq \gamma_{j}(j+1)$ for all $j \geq 0$

$$
\begin{aligned}
& \hat{\lambda} \gamma_{j}(j+1)^{2}-(j+1) \\
& =(j+1)\left(\gamma_{0}^{-1} \gamma_{j}(j+1)-1\right) \geq(j+1)(1-1)=0 .
\end{aligned}
$$

Thus $\mathcal{L}(f, \overline{\hat{\lambda}}) \geq 0$ for all $f \in X_{\gamma}$. Let $\hat{a}_{0}=\gamma_{0}^{-1 / 2}$ and $\hat{f}(z)=\hat{a}_{0}$ and notice $\|\hat{f}\|_{X}^{2}=1$ and clearly $\left|\hat{a}_{0}\right|^{2}=\gamma_{0}^{-1} \leq \delta_{0}^{2}$ so $\hat{f}$ is admissable in (31). Furthermore

$$
\mathcal{L}(\hat{f}, \overline{\hat{\lambda}})=\left|\hat{a}_{0}\right|^{2}\left(\hat{\lambda} \gamma_{0}-1\right)=0
$$


and so $\mathcal{L}(\hat{f}, \overline{\hat{\lambda}})=\min _{f \in X}(f, \overline{\hat{\lambda}})$. Also,

$$
\hat{\lambda}\left(\|\hat{f}\|_{X}^{2}-1\right)+\sum_{j=0}^{N-1} \hat{\lambda}_{j}\left(\frac{\left|\hat{a}_{j}\right|^{2}}{(j+1)^{2}}-\delta_{j}^{2}\right)=\hat{\lambda}(1-1)+0=0 .
$$

Therefore $E\left(W, K^{N}, \bar{\delta}\right)=\sqrt{\hat{\lambda}}=\gamma_{0}^{-1 / 2}$ and $\hat{m}(\tilde{y})=0$ is an optimal method.

The optimal method may not use all of the information provided as $p_{0}$ may be less than $N-1$. Thus increasing $N$ may not change $p_{0}$ and hence not change the error or the method. If $p_{0}<N-1$, then

$$
E\left(W, K^{p_{0}+1}, \bar{\delta}\right)=E\left(W, K^{N}, \delta\right)
$$

and we can reduce the amount of information needed for a given optimal error.

If $p_{0}=N-1$ we may be able to reduce the error of optimal recovery if we have more information available. Fix $\delta=\bar{\delta}_{\infty}=\left(\delta_{0}, \delta_{1}, \cdots\right)$. The greater number of terms we have of $K f$ then the better we may be able to approximate $f$, that is the smaller the optimal error of recovery. Let

$$
N_{\delta}=\max \left\{p \geq 0: \sum_{j=0}^{p} \delta_{j}^{2} \gamma_{j}(j+1)^{2} \leq 1\right\}
$$

and for $N_{\delta}<\infty$

$$
E\left(W, K^{N_{\delta}}, \delta\right) \leq E\left(W, K^{N}, \delta\right)
$$

for any $N \geq 0$. If we know the first $N_{\delta}$ terms with some errors, then further increasing the terms will not yield a decrease in the error of optimal recovery.

\subsection{Applications: The Hardy-Sobolev and Bergman-Sobolev Classes}

We now apply the general results to the Hardy-Sobolev and Bergman-Sobolev spaces of functions on the unit disc. Let $\mathcal{H}(\mathbb{D})$ denote the set of functions holomorphic on the unit disc. Define the Hardy space of functions $\mathcal{H}^{2}(\mathbb{D})$ as the set of all $f \in \mathcal{H}(\mathbb{D}), \quad f(z)=\sum_{j=0}^{\infty} a_{j} z^{j}$ with $\|f\|_{\mathcal{H}^{2}(\mathbb{D})}<\infty$ where

$$
\|f\|_{\mathcal{H}^{2}(\mathbb{D})}=\sum_{j=0}^{\infty}\left|a_{j}\right|^{2} .
$$

The Hardy-Sobolev space of functions, $\mathcal{H}^{2, r}(\mathbb{D})$, are those $f \in \mathcal{H}(\mathbb{D})$ such that $f^{(r)} \in \mathcal{H}^{2}(\mathbb{D})$ and $H^{2, r}(\mathbb{D})$ is the class consisting of those $f \in \mathcal{H}^{2, r}(\mathbb{D})$ with $\left\|f^{(r)}\right\|_{\mathcal{H}^{2}(\mathbb{D})} \leq 1$. The Bergman space of functions $\mathcal{A}^{2}(\mathbb{D})$ is the space of all $f \in \mathcal{H}(\mathbb{D})$ such that

$$
\|f\|_{L_{2}(\mathbb{D})}^{2}=\int_{\mathbb{D}}|f(z)|^{2} \mathrm{~d} A<\infty .
$$

That is, $\mathcal{A}^{2}(\mathbb{D})$ is the space of all holomorphic functions in $L_{2}(\mathbb{D})$. The Bergman-Sobolev space of functions, $\mathcal{A}^{2, r}(\mathbb{D})$, consists of $f \in \mathcal{H}(\mathbb{D})$ with

$f^{(r)} \in \mathcal{A}^{2}(\mathbb{D})$ and $\mathcal{A}^{2, r}(\mathbb{D})$ as the class of all

$f \in \mathcal{A}^{2, r}(\mathbb{D})$ with $\left\|f^{(r)}\right\|_{L_{2}(\mathbb{D})} \leq 1$.

So each space can be considered as the space $X_{\gamma}$ with

$$
\gamma_{j}(X)=\left\{\begin{array}{cl}
0 & : j<r \\
\left(\frac{j !}{(j-r) !}\right)^{2} & : r \leq j, X=\mathcal{H}^{2, r}(\mathbb{D}) . \\
\left(\frac{j !}{(j-r) !}\right)^{2} \frac{1}{j-r+1} & : r \leq j, X=\mathcal{A}^{2, r}(\mathbb{D})
\end{array}\right.
$$

For each space of functions we have the collection of points $C_{X}=\left(\left\{\alpha_{j}, \beta_{j}\right\}\right)=\left(\left\{\gamma_{j}(j+1)^{2}, j+1\right\}\right)_{j \in \square}$. If $X=\mathcal{H}^{2, r}(\mathbb{D})$ then for $j \geq r$

$$
\gamma_{j}(j+1)^{2}=\left(\frac{j !}{(j-r) !}\right)^{2}(j+1)^{2}=\left(\frac{(j+1) !}{(j-r) !}\right)^{2} .
$$

Therefore for $r=0,1, \cdots$

$$
\lim _{j \rightarrow \infty}\left(\gamma_{j}(j+1)\right)^{-1}=\lim _{j \rightarrow \infty}\left(\frac{(j-r) !}{j !}\right)^{2} \frac{1}{j+1}=0 .
$$

In this case we consider the collection of points

$$
C_{\mathcal{H}^{2, r}}=\left\{(0,0) \cup\left\{\left(\gamma_{j}\left(\mathcal{H}^{2, r}\right)(j+1)^{2}, j+1\right)\right\}_{j \in \square}\right\} .
$$

It is easy to see that if $M=c o\left(C_{\mathcal{H}^{2, r}}\right)$ then the piecewise linear function $\theta(x)=\max \{y:(x, y) \in M\}$ will have points of break

$$
\left.\left\{(0, r) \cup\left\{\left(\frac{(j+1) !}{(j-r) !}\right)^{2}, j+1\right): j=r, r+1, \cdots\right\}\right\} .
$$

For the space $\mathcal{A}^{2, r}$, the points to consider are

$$
C_{\mathcal{A}^{2, r}}=\left\{(0,0) \cup\left\{\left(\gamma_{j}\left(\mathcal{A}^{2, r}\right)(j+1)^{2}, j+1\right)\right\}_{j \in \square}\right\} .
$$

Again let $\theta(x)=\max \left\{y:(x, y) \in \operatorname{co}\left(C_{A^{2, r}}\right)\right\}$ and thus the points of break of $\theta$ will be precisely 


$$
\left\{(0, r) \cup\left\{\left(\frac{\left(\frac{(j+1) !}{(j-r) !}\right)^{2}}{j-r+1}, j+1\right): j=r, r+1, \cdots\right\}\right\} \text {. }
$$

For the special case of $\mathcal{A}^{2,0}$, the function $\theta$ has only a single point of break at the origin as

$$
C_{\mathcal{A}^{2,0}}=\left\{(0,0) \cup\{(j+1, j+1)\}_{j \in \square}\right\}
$$

so that $\theta(x)=x$ for $x \geq 0$. Furthermore, $\mathcal{A}^{2,0}$ does not satisfy (7) as

$$
\lim _{j \rightarrow \infty}\left(\gamma_{j}\left(\mathcal{A}^{2,0}\right)(j+1)\right)^{-1}=\lim _{j \rightarrow \infty} \frac{1}{j+1}(j+1)=1 .
$$

Thus, in the applications of the general results, this case will be treated separately.

For notational purposes, let $\left(x_{s}, y_{s}\right)=\left(x_{s}(X), y_{s}(X)\right)$, $s=0,1, \cdots$ be the points of break of $\theta$ for the space $X$.

Corollary 1. Let $X=\mathcal{H}^{2, r}$ or $X=\mathcal{A}^{2, r}$. If $x_{s}<\delta^{-2} \leq x_{s+1}$ with $s>0$ or $r>0$ then the error of optimal recovery is given by (13) and (14) is an optimal method. If $s=0$ and $r=0$ then $E(W, K, \delta)=1$ and $\hat{m}(y)=0$ is optimal.

Proof. For the spaces $X=\mathcal{A}^{2, r}$ or $\mathcal{H}^{2, r}, y_{s}=0$ if and only if $s=0$ and $r=0$. Thus $y_{s}>0$ if and only if $s>0$ or $r>0$. Thus apply Theorem 3 to obtain the result for all spaces except $\mathcal{A}^{2,0}$. The dual problem in the case $X=\mathcal{A}^{2,0}$ leads to a simple Lagrange function. The dual problem is specifically

$$
\sum_{j=0}^{\infty} \frac{\left|a_{j}\right|^{2}}{j+1} \rightarrow \max , \quad \sum_{j=0}^{\infty} \frac{\left|a_{j}\right|^{2}}{j+1} \leq 1, \quad \sum_{j=0}^{\infty} \frac{\left|a_{j}\right|^{2}}{(j+1)^{2}} \leq \delta^{2} .
$$

Therefore the Lagrange function is simply given by

$$
\mathcal{L}\left(f, \lambda_{1}, \lambda_{2}\right)=\sum_{j=0}^{\infty} \frac{\left|a_{j}\right|^{2}}{(j+1)^{2}}\left(\lambda_{1}(j+1)+\lambda_{2}-(j+1)\right) .
$$

Now if we let $\hat{\lambda}_{1}=1$ and $\hat{\lambda}_{2}=0$ then $\mathcal{L}\left(f, \hat{\lambda}_{1}, \hat{\lambda}_{2}\right)=0$ for any $f \in X$. So now proceed as in Theorem 3 . As any $\hat{f} \in \mathcal{A}^{2,0}$ will minimize $\mathcal{L}$, choose $\hat{f}$ as in (18). The extremal problem (19) is solved similarly, and as $\hat{\lambda}_{2}=0$ then $a_{j}=0$ for $j=0,1, \cdots$.

It should be noted that the optimal method described is stable with respect to the inaccurate information data.

We now apply Theorem 4 to the Hardy-Sobolev spaces $\mathcal{H}^{2, r}$ and Bergman-Sobolev spaces $\mathcal{A}^{2, r}$ in which $s_{0}$ is explicitly defined to be the smallest nonnegative integer satisfying

$$
\frac{1}{\gamma_{N}(N+1)} \geq \frac{1}{\gamma_{s+1}(s+2)^{2}-\gamma_{s}(s+1)^{2}}
$$

For the case $W=A^{2,0}, \gamma_{j}(j+1)=1$ for all $j \geq 0$. Thus $s_{0}=0$ does not depend on $N$. So

$x_{s_{0}}\left(A^{2,0}\right)=x_{0}\left(A^{2,0}\right)=0$ and hence for any $\delta$ we are in the case $\delta^{-2}>x_{s_{0}}$.

Corollary 2. Let $X=\mathcal{H}^{2, r}$ or $X=\mathcal{A}^{2, r}$. Suppose $x_{s}<\delta^{-2} \leq x_{s+1}$ with $s<s_{0}$. If $s>0$ or $r>0$ then let $\hat{\lambda}_{1}, \hat{\lambda}_{2}$ be given by (12) and the optimal error is given by (13) and (23) is an optimal method. If $s=0$ and $r=0$ then $E\left(W, K^{N}, \delta\right)=1$ and $\hat{m}(\tilde{y})=0$ is an optimal method.

Otherwise suppose $\delta^{-2}>x_{s_{0}}$. If $s_{0}>0$ or $r>0$ then the optimal error is given by (13) and (23) is an optimal method with $\hat{\lambda}_{1}=\left(\gamma_{N}(N+1)\right)^{-1}$ and

$\hat{\lambda}_{2}=y_{s_{0}}-x_{s_{0}} \hat{\lambda}_{1}$. If $s_{0}=0$ and $r=0$ then

$E\left(W, K^{N}, \delta\right)=1$ and $\hat{m}(\tilde{y})=0$ is an optimal method.

Proof. As previously stated, if $X=\mathcal{A}^{2,0}$ the only break point of $\theta$ is $(0,0)$ and furthermore as $\gamma_{j}\left(\mathcal{A}^{2,0}\right)=(j+1)^{-1}$ then $l_{0}$ given by $(21)$ does not exist so we treat this special case. In this case, the dual extremal problem is

$$
\|f\|_{L_{2}(\mathbb{D})}^{2} \rightarrow \max , \quad \sum_{j=0}^{\infty} \frac{\left|a_{j}\right|^{2}}{j+1} \leq 1, \quad \sum_{j=0}^{N-1} \frac{\left|a_{j}\right|^{2}}{(j+1)^{2}} \leq \delta^{2}
$$

and the corresponding Lagrange function is simply

$$
\mathcal{L}\left(f, \lambda_{1}, \lambda_{2}\right)=\sum_{j=0}^{\infty} \frac{\left|a_{j}\right|^{2}}{(j+1)^{2}}\left(\lambda_{1}(j+1)+\chi_{j}^{N} \lambda_{2}-(j+1)\right) .
$$

If $\hat{\lambda}_{1}=1$ and $\hat{\lambda}_{2}=0$ then $\mathcal{L}(f, 1,0)=0$ for any $f \in A^{2,0}$. Now proceed as in the proof of Theorem 4 to obtain the result.

We now apply Theorem 5 to the spaces $X=\mathcal{H}^{2, r}$ or $X=\mathcal{A}^{2, r}$ for $0 \leq r \leq N-1$. In this situation $\gamma_{j}(j+1)$ will be a non-decreasing sequence for all $j \geq 0$. Also, for any $r>0$ we have $\gamma_{0}=0$ and we are always in the case $\gamma_{0} \delta_{0}^{2} \leq 1$. For $r=0$ then for both the Hardy and Bergman spaces $\gamma_{0}=1$ and so the condition $\gamma_{0} \delta_{0}^{2} \leq 1$ will be satisfied if we know $\tilde{y}_{0}$ satisfying

$$
\left|a_{0}-\tilde{y}_{0}\right|=\left|K_{0} f-\tilde{y}_{0}\right| \leq \delta_{0} \leq 1 \text {. }
$$

Corollary 3. Let $X=\mathcal{H}^{2, r}$ or $X=\mathcal{A}^{2, r}$ with $1 \leq r \leq N-1$ or $r=0$ and $\delta_{0} \leq 1$ and $p_{0}$ given by (27). Let $\hat{\lambda}, \hat{\lambda}_{j}, j=0, \cdots, N-1$ be given by (28). Then the error of optimal recovery is given by (29) and (38) is an optimal method. If $r=0$ and $\delta_{0}>1$ then $E\left(W, K^{N}, \bar{\delta}\right)=1$ and $\hat{m}(\tilde{y})=0$ is an optimal method.

Proof. For Theorem 5 we simply used conditions (6) and (20), both of which are satisfied by $\mathcal{H}^{2, r}$ and $\mathcal{A}^{2, r}$ 
for all $0 \leq r \leq N-1$.

As a direct consequence of Theorem 5, we consider the situation in which we have a uniform bound on the inaccuracy of each of the first $N$ terms of $K_{j} f$. That is we take $\delta_{j}=\delta$ for every $0 \leq r \leq N-1$. If $\delta^{2} \gamma_{0} \leq 1$ we define $p_{0}$ similarly as

$$
p_{0}=\max \left\{p \geq 0: \delta^{2} \sum_{j=0}^{p} \gamma_{j}(j+1)^{2} \leq 1, \quad p \leq N-1\right\}
$$

and the apriori information is given by the values $\tilde{y}_{0}, \tilde{y}_{1}, \cdots, \tilde{y}_{N-1}$ such that

$$
\left|K_{j} f-\tilde{y}_{j}\right| \leq \delta .
$$

Again we will only need the values $\tilde{y}_{0}, \cdots, \tilde{y}_{p_{0}}$ for an optimal method.

As previously noted, since the optimal method and error of optimal recovery only use up to the $p_{0}$ term then any information beyond may be disregarded if $p_{0}<N-1$ as additional information will not decrease the error of optimal recovery.

\section{REFERENCES}

[1] K. Y. Osipenko, "Optimal Recovery of Linear Operators from Inaccurate Information,” MATI-RSTU, Department of Mathematics, Washington DC, 2007, pp. 1-87.

[2] G. G. Magaril-Il'yaev and K. Y. Osipenko, "Optimal Recovery of Functions and Their Derivatives from Fourier Coefficients Prescribed with an Error," Sbornik: Mathematics, Vol. 193, No. 3, 2002, pp. 387-407.
[3] G. G. Magaril-Il'yaev and K. Y. Osipenko, "Optimal Recovery of Functions and Their Derivatives form Inaccurate Information about the Spectrum and Inequalities from Derivatives," Functional Analysis and Its Applications, Vol. 37, No. 3, 2003, pp. 203-214. doi:10.1023/A:1026084617039

[4] A. G. Marchuk and K. Y. Osipenko, "Best Approximations of Functions Specified with an Error at a Finite Number of Points," Mathematical Notes of the Academy of Sciences of the USSR, Vol. 17, No. 3, 1975, pp. 207212. doi:10.1007/BF01149008

[5] A. A. Melkman and C. A. Micchelli, "Optimal Estimation of Linear Operators in Hilbert Spaces from Inaccurate Data," SIAM Journal on Numerical Analysis, Vol. 16, No. 1, 1979, pp. 87-105. doi:10.1137/0716007

[6] K. Y. Osipenko, "Best Approximation of Analytic Functions from Information about Their Values at a Finite Number of Points," Mathematical Notes of the Academy of Sciences of the USSR, Vol. 19, No. 1, 1976, pp. 17-23. doi:10.1007/BF01147612

[7] K. Y. Osipenko, "The Hardy-Littlewood-Polya Inequality for Analytic Functions from Hardy-Sobolev Spaces," Sbornic: Mathematics, Vol. 197, No. 3, 2006, pp. 315334.

[8] K. Y. Osipenko and M. I. Stessin, "Hadamard and Schwarz Type Theorems and Optimal Recovery in Spaces of Analytic Functions," Constructive Approximation, Vol. 31, No. 1, 2009, pp. 37-67.

[9] K. Y. Osipenko and N. D. Vysk, "Optimal Recovery of the Wave Equation Solution by Inaccurate Input Data," Matematicheskie Zametki, Vol. 81, No. 6, 2007, pp. 723733. 